\title{
99. Einheitliche Theorie der Funktionen einer binären komplexen Veränderlichen, $2^{1}$.
}

\author{
Von Tsurusaburo TAKasU. \\ Mathematisches Institut der Tohoku Kaiserlichen Universität, Sendai. \\ (Comm. by T. YoSIE, M.I.A., Dec. 12, 1941.)
}

Im I. Abschnitt ${ }^{2}$ habe ich die Grundbegriffe der Theorie der Funktionen einer binären komplexen Veränderlichen $z=x+j y,\left(j^{2}=\mu+\nu j ; \mu, \nu, x, y\right.$ : reelle Zahlen) skizziert. Im Folgenden möchte ich (i) drei Beweisweisen des Analogons der Cauchyschen Integralformel angeben, (ii) das Analogon des Moreraschen Satzes beweisen, und dann (iii) drei verbesserte Beweise der Beltrami-Pompéiuschen Integralformel ausführen.

1. Bezeichnungen.

$$
\begin{gathered}
z=x+j y=\rho(\operatorname{cosj} \theta+j \operatorname{sinj} \theta)=\rho e^{j \theta}, \\
\bar{z}=x+\bar{j} y=\rho(\operatorname{cosj} \theta+\bar{j} \operatorname{sinj} \theta)=\rho e^{-j \theta}, \\
\rho^{2}=z \bar{z}=x^{2}+\nu x y-\mu y^{2}, \quad \rho=\|z\|=\|\bar{z}\|, \\
\operatorname{cosj}^{2} \theta+\nu \operatorname{cosj} \theta \operatorname{sinj} \theta-\mu \operatorname{sinj}^{2} \theta=1, \\
\operatorname{sinj} \theta=\frac{e^{j \theta}-e^{-j \theta}}{2 j-\nu}, \quad \operatorname{cosj} \theta=\frac{j\left(e^{j \theta}+e^{-j \theta}\right)-\nu e^{j \theta}}{2 j-\nu} .
\end{gathered}
$$

Das Zeichen $|z|$ bedeute im Falle $\nu^{2}+4 \mu$

$$
<0 \quad \rightarrow 0 \quad \mid>0
$$

den absoluten Betrag den absoluten Betrag des Segmentes, welches im gewöhnlichen Sinne: die isotrope Gerade $(X-x)+j(Y-y)=0$ aus $|z|=|\|z\||=|\|\bar{z}\||$. der $x$-Achse ausschneidet.

2. Ein Analogon zum Cauchyschen Integalsatz. Die Formel

$$
\int_{C} f(z) d z=0
$$

für analytische Funktion $f(z)$ und für einfachgeschlossene Kurve $C$ ist eine unmittelbare Folge der Greenschen Formel und der verallgemeinerten Cauchy-Riemannschen Gleichungen : $u_{1} \equiv \mu v_{x}-u_{y}=0, v_{1} \equiv u_{x}+\nu v_{x}-u_{y}=0$, und ist in der Formel

1) Dieses Stück gehört zur Reihe von Untersuchungen, welche auf Kosten der Ausgaben des Unterrichtsministeriums für wissenschaftlichen Forschung ausgeführt werden.

2) Proc. 17 (1941), 349-357. Zur dortigen Literaturliste möchte ich die Folgenden hinzufügen :

M. Futagawa, On the Theory of a Quaternary Variable. Tohoku Math. Journ., 20 (1928), 173-222. 69-120.

M. Futagawa, On the Theory of a Quaternary Variable (Part II). Ibid., 35 (1932),

F. Ringleb, Beiträge zur Funktionentheorie in komplexen Systemen I. Rend. Palermo, 57 (1933), 311-350. 
(2)

$$
\int_{C} f(z) d z=\iint_{C} D f(\zeta) d \xi d \eta, \quad D f(z)=u_{1}+j v_{1}
$$

von Herrn J. Rey Pastor ${ }^{3)}$ enthalten.

3. Drei Beweisweisen fürs Analogon der Cauchyschen Integralformel :

(3)

$$
f(z)=\frac{1}{2 \pi i} \int_{C} \frac{f(\zeta)}{\zeta-z} d \zeta .
$$

Erster Beweis. Man betrachte im Falle $\nu^{2}+4 \mu$

die Ellipse :

$$
\begin{aligned}
& \rightarrow 0 \text { das isotrope } \\
& \text { Parallelenpaar : }
\end{aligned}
$$

$$
|\|\zeta-z\||=k
$$

mit genügend kleinem $k>0$. Die einfachgeschlossene Kurve $C$ schiesst diese Ellipse trifft diese ein, $\quad$ Parallelen,

Hyperbeln,

da $k$ genügend klein angenommen ist. Den ausserhalb der Ellipse

Parallelen

Hyperbeln

$$
P: \quad|\|\zeta-z\||=k
$$

liegenden Bereich von $C$ bezeichnen wir mit $C_{k}-P_{k}$, wobei $C_{k}$ den dabei in Betracht kommenden Teilen von $C$ entspricht.

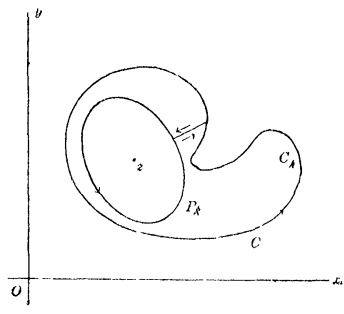

Fig. 1.

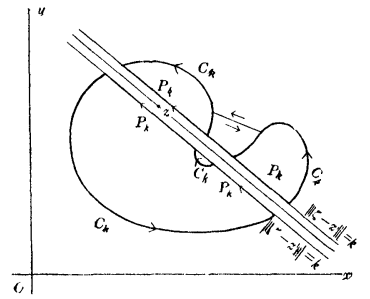

Fig. 2 .

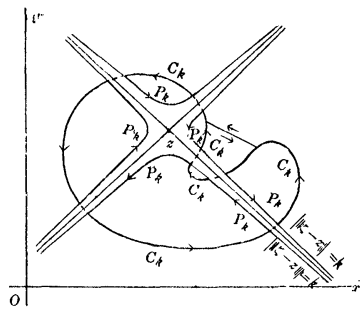

Fig. 3.

Auf die Funktion $f(\zeta):(\zeta-z)$ im Bereich $C_{k}-P_{k}$ ist die Formel (1) anwandbar, da kein Nullteiler darin liegt:

$$
\begin{aligned}
& \int_{C_{k}-P_{k}} \frac{f(\zeta)}{\zeta-z} d \zeta=0 \quad \text { d. h. } \int_{C_{k}+P-P_{k}}=\int_{P_{k}+P-P_{k}}=\int_{P}, \\
& \int_{P}=\int_{P} f\left(z+\rho e^{j \theta}\right)\left(\frac{d|\rho|}{|\rho|}+j d \theta\right), \quad(|\rho|=k) \\
& =j \int_{P} f\left(z+k e^{j \theta}\right) d \theta \rightarrow j f(z) 2 \pi \frac{i}{j},{ }^{4)} \quad(k \rightarrow 0),
\end{aligned}
$$

3) J. Rey Pastor, Funciones de Variable Compleja Binaria, Boletin del Seminario Matemático, Buenos Aires, 19 (1936), 101-116.

4) Es ist überhaupt $\oint d \theta=2 \pi \frac{i}{j}$ auf Grund der $j$-Orthogonalinvolution, selbst wenn $1: \zeta-z$ nicht beschränkt ist. 
da $f(\zeta)$ stetig ist. Daher ist $\left(C_{k}+P-P_{k} \rightarrow C\right)$ :

$$
\int_{C} \frac{f(\zeta)}{\zeta-z} d \zeta=2 \pi i f(z)
$$

Zweiter Beweis. (Diese Beweisweise kehrt im Falle $\nu^{2}+4 \mu<0$ zur letzten zurück.) Man betrachte im Falle $\nu^{2}+4 \mu$

$<0$ die Ellipse:

$$
|\zeta-z|=k
$$

$$
>0
$$

das isotrope Parallelenpaar $(\zeta-z)^{2}=k^{2}$

mit genügend kleinem $k>0$. Die einfachgeschlossene Kurve $C$

schliesst dann diese

Ellipse ein. (Fig. 1.) trifft dann dieses Parallelenpaar.

(Ungefähr Fig. 2.)

Den ausserhalb der

Ellipse $P:|\zeta-z|=k \mid$ Geraden $P: \quad(\zeta-z)^{2}=k^{2}$

liegenden Bereich von $C$ bezeichnen wir mit $C_{k}-P_{k}$, wobei $C_{k}$ den dabei in Betracht kommenden Teilbogen von $C$ entspricht. Auf die Funktion $f(\zeta):(\zeta-z)$ im Bereich $C_{k}-P_{k}$ ist die Formel (1) anwendbar, da kein Nullteiler darin liegt:

$$
\begin{aligned}
& \int_{C_{k}-P_{k}} \frac{f(\zeta)}{\zeta-z} d \zeta=0 \text { d.h. } \int_{C_{k}+P-P_{k}}=\int_{P_{k}+P-P_{k}}=\int_{P} \text {, } \\
& \int_{P}=\int_{P} f\left(z+k e^{j \theta}\right) \quad\left|\int_{P}=\int_{(\zeta-z)^{2}=k^{2}} f(\zeta) d \log \right| \zeta-z \mid+\int_{\zeta-z=k \rightarrow \zeta-z=-k} f(z+k) d \log (\zeta-z) \\
& \times\left(\frac{d k}{k}+j d \theta\right) \quad+\int_{\substack{\zeta-z=-k \rightarrow \zeta-z=k \\
f}}^{f(z-k) d \log (\zeta-z)} \\
& \begin{aligned}
\rightarrow f(z)\left(0+2 \pi \frac{i}{j} j\right), & \rightarrow 0+f(z) \log e^{\pi i}+f(z) \log e^{\pi i}, \quad(k \rightarrow 0), \\
(k \rightarrow 0), &
\end{aligned} \\
& \int_{C} \frac{f(\zeta)}{\zeta-z} d \zeta=2 \pi i f(z), \quad(k \rightarrow 0)
\end{aligned}
$$

Dritter Beweis. Ergänzt man die geschlossene Kurve $C$ mit dem Kreise $K_{r}:(X-x)^{2}+(Y-y)^{2}=r^{2}$, so gilt der Cauchy-Pastorsche Satz:

$$
\int_{C-K_{r}} \frac{f(\zeta)}{\zeta-z} d \zeta=0, \text { d. h. } \int_{C}=\int_{K_{r}},
$$

wobei ist:

$$
\begin{aligned}
\int_{K_{r}}=\int_{K_{r}} f(\zeta) d \log (\zeta-z)=\int_{K_{r}} f(z+r(\cos \varphi+j \sin \varphi)) \\
\quad \times\left\{\frac{d r}{r}+d \log (\cos \varphi+j \sin \varphi)\right\} \\
=\int_{K_{r}} f(z+r(\cos \varphi+j \sin \varphi))(d \log |\cos \varphi+j \sin \varphi|+*) .
\end{aligned}
$$

Dabei bedeutet das Zeichen * den Beitrag aus den zwei Stellen, wo der Ausdruck $\cos \varphi+j \sin \varphi$ ihr Vorzeichen ändert. Also ist 


$$
\int_{K_{r}} \rightarrow f(z)\left(0^{5)}+2 \log e^{\pi i}\right)=2 \pi i f(z), \quad(r \rightarrow 0),
$$

da $\int_{C}$ von $r$ unabhängig ist. Daher ist

$$
\int_{C} \frac{f(\zeta)}{\zeta-z} d \zeta=2 \pi i f(z)
$$

Zusatz.

$$
\int_{C} \frac{d \zeta}{\zeta-z}=2 \pi i
$$

4. Analytischer Charakter eines Integrals. Das in der Integralformel vorkommende Integral

$$
J(z)=\int_{C} \frac{f(\zeta)}{\zeta-z} d \zeta
$$

stellt in einem einen Kurvenbogen $C$ nicht enthaltenden Bereich eine analytische Funktion von $z$ dar, wenn $C$ ein beliebiger, die Isotrope $\zeta-z=0$ nicht treffender, j-rektifizierbarer Kurvenbogen ist, auf welchem $f(\zeta)$ stetig ist. Unter $z$ ist dabei eine beliebige nicht auf der Kurve $C$ gelegene Stelle zu verstehen.

Man findet nämlich dann für den Differenzenquotienten den Ausdruck

$$
\frac{J(z+h-J(z)}{h}=\int_{C} \frac{f(\zeta) d \zeta}{(\zeta-z)^{2}(\zeta-z-h)}, \quad(|h| \neq 0) .
$$

Man wird vermuten, dass dieser Ausdruck für $h \rightarrow 0$ dem Werte $\int_{C} \frac{f(\zeta)}{(\zeta-z)^{2}} d \zeta$ zustrebt, und in der Tat ist ja auch

$$
\frac{J(z+h)-J(z)}{h}-\int_{C} \frac{f(\zeta)}{(\zeta-z)^{2}} d \zeta=\int_{C} f(\zeta) \frac{h}{(\zeta-z)^{2}(\zeta-z-h)} d \zeta
$$

Also wird

$$
\left|\frac{J(z+h)-J(z)}{h}-\int_{C} \frac{f(\zeta)}{\zeta-z} d \zeta\right| \leqq \frac{\operatorname{Max}|f(\zeta)| \cdot|h| \cdot L}{d^{3}},(i:(j, \bar{j})),
$$

wenn $L$ die $j$-Länge der Kurve $C$ und $d$ das $\operatorname{Min}(|\zeta-z|,|\zeta-z-h|)$ bedeutet. Daher ist in der Tat

(8) $\quad \lim _{h \rightarrow 0} \frac{J(z+h)-J(z)}{h}=\int_{C} \frac{f(\zeta)}{(\zeta-z)^{2}} d \zeta, \quad(|h| \neq 0), \quad(j:(j, \bar{j}))$

und $J(z)$ ist wirklich eine analytische Funktion von $z$ in der Umgebung einer jeden Stelle, die nicht auf der Kurve $C$ liegt.

5. Integralformel für Ableitungen einer analytischen Funktion. In einem einfachzusammenhängenden Bereich $B$ sei die Funktion $f(z)$ eindeutig und analytisch. $C$ sei eine geschlossene, in $B$ verlaufende

5) Genauer ein Grenzprozess mit Heranziehung der $j$-Orthogonalinvolution! 
$j$-rektifizierbare Kurve, welche $\zeta=z$ einmal im positiven Sinne umläuft. Dann ist ${ }^{6}$

$$
f^{(n)}(z)=\frac{n !}{2 \pi i} \int_{C} \frac{f(\zeta)}{(\zeta-z)^{n+1}} d \zeta .
$$

Zusatz $1^{\circ}$. Eine jede analytische Funktion kann beliebig oft differenzierbar werden. Die Ableitungen aller Ordnungen sind also wieder analytische Funktionen.

Zusatz $2^{\circ}$. Aus der Differenzierbarkeit einer Funktion folgt auch die Stetigkeit der Ableitung.

6. Umkehrung der verallgemeinerten Cauchyschen IntegralsatzesVerallgemeinerung des Moreraschen Satzes.

Hilfssatz. Jede in einem einfachzusammenhängenden Bereiche eindeutige analytische Funktion $f(z)$ besitzt ein Integral, d.h. es gibt bis auf eine analytische Funktion

$$
J(z)=\int_{0}^{z} f(\zeta) d \zeta
$$

die gleichfalls im Bereiche $B$ eindeutig ist und deren Ableitung $f(z)$ ist.

Beweis. Einen Weg, über den das obige Integral zu erstrecken ist, brauchen wir nun nicht mehr anzugeben, denn folgt aus dem nun bewiesenen verallgemeinerten Cauchyschen Integralsatz, dass der Wert des Integrals davon unabhängig ist, über welchen in $B$ gelegenen Weg man es erstreckt. Das Integral $J(z)$ stellt also eine in $B$ eindeutige Funktion dar. Wir zeigen, dass $J(z)$ analytisch ist und dass $f(z)$ ihre Ableitung ist. Es wird nämlich

$$
\frac{J(z)-J\left(z_{0}\right)}{z-z_{0}}-f\left(z_{0}\right)=\frac{\int_{z_{0}}^{z}\left[f(\zeta)-f\left(z_{0}\right)\right] d \zeta}{z-z_{0}}, \quad\left(\left|z-z_{0}\right| \neq 0\right) .
$$

Da aber $f(z)$ bei $z_{0}$ stetig ist, so gibt es eine Funktion $\delta(\varepsilon)$, so dass

$$
\left|f(\zeta)-f\left(z_{0}\right)\right|<\varepsilon \text { ist für } 0<\left|\zeta-z_{0}\right|<\delta(\varepsilon), \quad(j:(j, \bar{j})) .
$$

Da weiter der Integrationsweg von $z_{0}$ bis $z$ geradlinig gewählt werden darf, so ist seine $j$-Länge $\left|z-z_{0}\right|$. Daher wird

$$
\begin{gathered}
\int_{z_{0}}^{z}\left[f(\zeta)-f\left(z_{0}\right)\right] d \zeta<\varepsilon\left|z-z_{0}\right|, \quad(j:(j, j)), \\
\left|\frac{J(z)-J\left(z_{0}\right)}{z-z_{0}}-f\left(z_{0}\right)\right|<\varepsilon \text { für } 0<\left|z-z_{0}\right|<\delta(\varepsilon), \quad(j:(j, j)) .
\end{gathered}
$$

Also ist $J(z)$ analytisch und $f(z)$ ist seine Ableitung.

Verallgemeinerung des Moreraschen Satzes. Eine in einem Bereiche $B$ eindeutige und stetige Funktion $f(z)$, für welche das Integral $\int_{C} f(z) d z$ verschwindet, ganz einerlei, über welche dem Bereich angehörige geschlossene Kurve $C$ es auch erstreckt wird, ist notwendig eine analytische Funktion.

6) Hierbei ist ein Grenzprozess $\lim _{k \rightarrow 0} C_{k} \rightarrow C$ im Sinne der Nr. 3 benutzt. 
Beweis. Das Integral $J(z)=\int_{0}^{z} f(\zeta) d \zeta$ stellt eine im Bereiche eindeutige Funktion dar, weil das Integral vom Wege unabhängig ist. Diese Funktion ist aber analytisch. Denn schon im Hilfssatz gezeigt, dass $J(z)$ differenzierbar ist. Es wird nach der dort angestellten Überlegung $J^{\prime}(z)=f(z)$. Im Zusatz $1^{\circ}$ letzter $\mathrm{Nr}$. aber haben wir gesehen, dass, die Ableitung einer analytischen Funktion selbst analytisch ist. Also ist $f(z)$ wirklich analytisch.

7. Drei Beweisweisen der Beltrami-Pompéiuschen Integralformel. Es sei $f(z)=u(x, y)+j v(x, y)$ eine eindeutige, stetige mit stetigen $u_{x}, u_{y}$, $v_{x}, v_{y}$ versehene, in einem Bereich $B$ definierte Funktion derart, dass ihre Flächenderivierte

$$
\left.\frac{d f}{d A} \equiv D f=\left(\mu v_{x}-u_{y}\right)+j\left(u_{x}+\nu v_{x}-v_{y}\right) \equiv \frac{\partial f}{\partial \bar{z}} 7\right)
$$

existiert. Dann gilt die Formel :8)

$$
f(z)=\frac{1}{2 \pi i} \int_{C} \frac{f(\zeta)}{\zeta-z} d \zeta-\frac{1}{2 \pi i} \iint_{C} \frac{D f(\zeta)}{\zeta-z} d \xi d \eta, \quad(\zeta=\xi+j \eta)
$$

Dabei ist $C$ eine beliebige in $B$ gelegene einfachgeschlossene Kurve.

Erster Beweis. Die Funktion

$$
F(\zeta)=\frac{f(\zeta)}{\zeta-z}=P(\xi, \eta)+j Q(\xi, \eta), \quad(|\zeta-z| \neq 0)
$$

ist eine eindeutige, stetige, mit stetigen $P_{x}, P_{y}, Q_{x}, Q_{y}$ versehene, im Bereiche $B$ definierte Funktion derart, dass ihre Flächenderivierte

$$
\frac{d F(\zeta)}{d A}=\frac{\partial F(\zeta)}{\partial \zeta}=\frac{\partial f(\zeta)}{\partial \bar{\zeta}}:(\zeta-z)
$$

existiert. Dabei ist $z$ irgend ein Punkt in $B$. Betrachten wir mit einem genügend kleinen $k>0$

\begin{tabular}{l|l|l} 
die Ellipse der Fig. 1: & $\begin{array}{l}\text { das isotrope Parallelen- } \\
\text { paar der Fig. 2: }\end{array}$ & $\begin{array}{l}\text { die konjugierten } \\
\text { Hyperbeln der Fig. 3: }\end{array}$
\end{tabular}

$$
P_{k}: \quad|\|\zeta-z\||=k .
$$

Dann enthält der Bereich $C_{k}-P_{k}$ keine Nullteiler. Wendet man die Formel (2) auf die Funktion $F(\zeta)$ an, so ergibt sich:

$$
\int_{C_{k_{k}-P_{k}}} \frac{f(\zeta)}{\zeta-z} d \zeta=\iint_{C_{k}-P_{k}} \frac{D f(\zeta)}{\zeta-z} d \xi d \eta
$$

Nun ist

7) Dies folgt aus: $\left(\frac{d f}{d z}\right)=\frac{\partial f}{\partial z}+\frac{d f}{d A} e^{-2 j \varphi}$ und $\left(\frac{d f}{d z}\right)=\frac{\partial f}{\partial z}+\frac{\partial f}{\partial \bar{z}} \frac{d \bar{z}}{d z}=\frac{\partial f}{\partial z}$ $+\frac{\partial f}{\partial \bar{z}} e^{-2 j \varphi}$.

8) Bei Herrn J. Rey Pastor ist die Formel (11) nur zum Falle $j^{2}=-1$ beschränkt. Die hiesigen Beweisweisen schliessen sich aber teilweise an seine Idee an. 


$$
\begin{aligned}
\int_{C_{k}-P_{k}} \frac{f(\zeta)}{\zeta-z} d \zeta= & \int_{C_{k}+P-P_{k}} \frac{f(\zeta)}{\zeta-z} d \zeta-\int_{P_{l_{k}+P-P_{k}}} f\left(z+\rho e^{j \theta}\right)\left(\frac{d|\rho|}{|\rho|}+j d \theta\right), \quad(|\rho|=k) \\
& \rightarrow \int_{C} \frac{f(\zeta)}{\zeta-z} d \zeta-2 \pi i f(z), \quad(k \rightarrow 0) .
\end{aligned}
$$

Also existiert:

$$
\iint_{C} \frac{D f(\zeta)}{\zeta-z} d \xi d \eta=\lim _{k \rightarrow 0} \iint_{C_{k}-P_{l}} \frac{D f(\zeta)}{\zeta-z} d \xi d \eta=\int_{C} \frac{f(\zeta)}{\zeta-z} d \zeta-2 \pi i f(z) .
$$

$\nu^{2}+4 \mu$

$<0$ die Ellipse der

Fig. 1: $|\zeta-z|=k$.

$\rightarrow 0 \quad \quad>0$

Den ausserhalb der

Ellipse $P:|\zeta-z|=k \mid$ Geraden $P: \quad(\zeta-z)^{2}=k^{2}$

liegenden Bereich von $C$ mit $C_{k}-P_{k}$ wie in Nr. 3. Dann enthält $C_{k}-P_{k}$ keine Nullteiler. Wendet man die Formel (2) auf die Funktion $F(\zeta)$ an, so ergibt sich:

$$
\int_{C_{k_{k}-P_{k}}} \frac{f(\zeta)}{\zeta-z} d \zeta=\iint_{C_{k_{k}-P_{k}}} \frac{D f(\zeta)}{\zeta-z} d \zeta d \eta \text {. }
$$

Nun ist

$$
\begin{array}{cc} 
& \int_{C_{k}-P_{k}} \frac{f(\zeta)}{\zeta-z} d \zeta=\int_{C_{k}+P-P_{k}} \frac{f(\zeta)}{\zeta-z} d \zeta \\
-\int_{P_{k}+P-P_{k}} f\left(z+k e^{j \theta}\right) & -\int_{(\zeta-z)^{2}-k^{2}} f(\zeta) d \log |\zeta-z|-\int_{\zeta-z=k \rightarrow \zeta-z=-k} f(z+k) d \log (\zeta-z) \\
\times\left(\frac{d k}{k}+j d \theta\right) & -\int_{\zeta-z=-k \rightarrow \zeta-z-k} f(z-k) d \log (\zeta-z) \\
\rightarrow \int_{C}+f(z)\left(0+2 \pi \frac{i}{j} j\right) & \rightarrow 0-f(z) \log e^{\pi i}-f(z) \log e^{\pi i} \\
= & \int_{C} \frac{f(\zeta)}{\zeta-z} d \zeta-2 \pi i f(z), \quad(k \rightarrow 0) .
\end{array}
$$

Also existiert :

$$
\iint_{C} \frac{D f(\zeta)}{\zeta-z} d \xi d \eta=\lim _{k \rightarrow 0} \iint_{C_{l_{k}-P_{k}}} \frac{D f(\zeta)}{\zeta-z} d \xi d \eta=\int_{C} \frac{f(\zeta)}{\zeta-z} d \zeta-2 \pi i f(z) .
$$

Dritter Beweis. Genau wie beim dritten Beweis der Nr. 3 kann man den Kreis zum jetzigen Zwecke benutzen.

8. Schlussbemerkungen. (i) In meiner Theorie tut man nicht nur mit dem Ring von $z=x+j y$, sondern auch mit den nichteuklidischen 


\begin{tabular}{l|l|l}
$\begin{array}{l}\text { EE-Geom., } \\
\text { HE-Geom. und }\end{array}$ & $\begin{array}{c}\text { EP-Geom., } \\
\text { HP-Geom. und } \\
\text { PP-Geom., }\end{array}$ & $\begin{array}{l}\text { EH-Geom., } \\
\text { HH-Geom. und } \\
\text { PH-Geom., }\end{array}$ \\
$\begin{array}{l}\text { in welchen die Winkelmassbestimmung } \\
\text { elliptisch }\end{array}$ & parabolisch
\end{tabular}

ist, so dass die gewöhnliche imaginäre Einheit $i\left(i^{2}=-1\right)$ als Hilfsmittel ins Winkelmass hineintritt, was aber das $\mathrm{zu}$ Grunde liegende eigentliche Komplexzahlensystem gar nicht zerstört (etwa wie Öl im Wasser!). (ii) In den beiden Fällen $\nu^{2}+4 \mu \rightarrow 0$ und $\nu^{2}+4 \mu>0$ ist der Modul $\|z\|$ vom absoluten Betrag $|z|$ unterschieden. (iii) Im Grenzbegriff $\lim _{z \rightarrow a} f(z)=A$ ist die Zweideutigkeit $(j:(j, \bar{j}))$ von $j$ wesentlich. (iv) Also sind Ableitung und Stetigkeit von den Fällen $\nu^{2}+4 \mu \rightarrow 0$ und $\nu^{2}+4 \mu>0$ von denjenigen im Reellen darum verschieden, dass die Zweideutigkeit von $j$ von selbst in Betracht kommt. (v) Die Formeln (3) und (11) sind notwendige Folgen von der Zweidimensienalität von $z$ und stammt aus der Periodizität der Funktion log. (vi) Die Formel $\oint d \theta=2 \pi \frac{i}{j}$, welche aus der logarithmischen Definition des Winkels (also aus der Natur der Funktion $\log z$ ) sowie aus der $j$-Orthogonalinvolution stammt, ist für den Beweis der Integralformeln (3) und (11) zu Grunde gelegt.

Berichtigung zum I. Abschnitt (Proc. 17, 1941) :

\begin{tabular}{|c|c|c|c|}
\hline $\begin{array}{c}\text { Seite } \\
351\end{array}$ & $\begin{array}{l}\text { Linie } \\
20\end{array}$ & $\begin{array}{r}\text { für } \\
\operatorname{sinj}^{2} \theta-\end{array}$ & $\operatorname{sinj} \theta-$ \\
\hline 352 & 15 & $d x d y=\cdots \rho d \rho d \theta$ & $\iint d x d y=\cdots \iint \rho d \rho d \theta$ \\
\hline " & 26 & $\left(z_{n}-z \ldots 0\right)$ & $(j:(j, \bar{j}))$ \\
\hline 353 & 4 & $\sqrt[n]{a_{n}}$ & $\sqrt[n]{\left|a_{n}\right|},(j:(j, \bar{j}))$ \\
\hline " & 6 & $(f(z) \ldots 0)$ & $(j:(j, \bar{j}))$ \\
\hline$\ddot{~}$ & $\begin{array}{l}\quad 26 \\
1 \text { v. unten } \\
\text { Fussnote }\end{array}$ & $\begin{array}{l}\text { Nr. } 8, \text { rechts } \\
\text { eine...Kurve } \\
\text { "rechte Seite" }\end{array}$ & $\begin{array}{l}\text {, nur den Fall } D f=0 \text { “ } \\
\text { der Rand von } D \\
\text {, nichts" }\end{array}$ \\
\hline$”$ & 2 & $\iint$ & $\iint_{A}$ \\
\hline " & 6 & $G$ & $C$ \\
\hline$"$ & $"$ & $\begin{array}{r}D \\
d z\end{array}$ & ${ }_{d z}^{A}$ \\
\hline$"$ & 8 & $\zeta-z$ & $\overline{z-\zeta}$ \\
\hline$"$ & $\begin{array}{l}5 \text { v. unten } \\
6 \text { v. unten }\end{array}$ & $\begin{array}{l}\text { mit...G } \\
D\end{array}$ & $\begin{array}{l}\text { dem Rand } G \text { von } D \\
A\end{array}$ \\
\hline 356 & 2 & ist nach (7) & ist \\
\hline$"$ & 12 & $\zeta-$ & $C-$ \\
\hline$"$ & 16 & $\int_{G}^{*} \frac{f(z)}{z-\zeta} d z+\cdots d z$ & $\int_{G}^{*} \frac{f(z)}{z-\zeta} d z-\int_{(\zeta, r)}^{*} \frac{f(z)}{z-\zeta} d z$ \\
\hline " & 17 & $=\int_{G}^{*}+$ & $=\int_{G}^{*}-$ \\
\hline$"$ & 18 & Ist $\cdots=0$. & "nichts" \\
\hline
\end{tabular}

